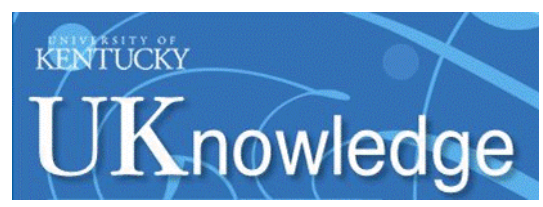

University of Kentucky

UKnowledge

\title{
Real-Time Sensing of Single-Ligand Delivery with Nanoaperture- Integrated Microfluidic Devices
}

\author{
W. Elliott Martin \\ University of Kentucky, william.martin7@uky.edu \\ Ning Ge \\ University of Kentucky, ning.ge@uky.edu \\ Bernadeta R. Srijanto \\ Oak Ridge National Laboratory \\ Emily Furnish \\ University of Kentucky, emily.furnish@uky.edu \\ C. Patrick Collier \\ Oak Ridge National Laboratory
}

See next page for additional authors

Follow this and additional works at: https://uknowledge.uky.edu/chemistry_facpub

Part of the Cell and Developmental Biology Commons, Chemistry Commons, Molecular Biology

Commons, and the Semiconductor and Optical Materials Commons

Right click to open a feedback form in a new tab to let us know how this document benefits you.

\section{Repository Citation}

Martin, W. Elliott; Ge, Ning; Srijanto, Bernadeta R.; Furnish, Emily; Collier, C. Patrick; Trinkle, Christine A.; and Richards, Christopher I., "Real-Time Sensing of Single-Ligand Delivery with Nanoaperture-Integrated Microfluidic Devices" (2017). Chemistry Faculty Publications. 97.

https://uknowledge.uky.edu/chemistry_facpub/97

This Article is brought to you for free and open access by the Chemistry at UKnowledge. It has been accepted for inclusion in Chemistry Faculty Publications by an authorized administrator of UKnowledge. For more information, please contact UKnowledge@lsv.uky.edu. 


\section{Real-Time Sensing of Single-Ligand Delivery with Nanoaperture-Integrated Microfluidic Devices}

\section{Digital Object Identifier (DOI)}

https://doi.org/10.1021/acsomega.7b00934

Notes/Citation Information

Published in ACS Omega, v. 2, issue 7, p. 3858-3867.

Copyright @ 2017 American Chemical Society

This is an open access article published under an ACS AuthorChoice License, which permits copying and redistribution of the article or any adaptations for non-commercial purposes.

\section{Authors}

W. Elliott Martin, Ning Ge, Bernadeta R. Srijanto, Emily Furnish, C. Patrick Collier, Christine A. Trinkle, and Christopher I. Richards 


\title{
Real-Time Sensing of Single-Ligand Delivery with Nanoaperture- Integrated Microfluidic Devices
}

\author{
W. Elliott Martin, ${ }^{\dagger, \|}$ Ning Ge, ${ }^{\ddagger} \|$ Bernadeta R. Srijanto, ${ }^{\S}$ Emily Furnish, ${ }^{\dagger}$ C. Patrick Collier, ${ }^{\S}$ \\ Christine A. Trinkle, ${ }^{*} *$ and Christopher I. Richards ${ }^{*}+\odot$ \\ ${ }^{\dagger}$ Department of Chemistry, University of Kentucky, 505 Rose Street, Lexington, Kentucky 40506, United States \\ ${ }^{\ddagger}$ Department of Mechanical Engineering, University of Kentucky, 151 Ralph G. Anderson Building, Lexington, Kentucky 40506, \\ United States \\ ${ }^{\S}$ Center for Nanophase Materials Sciences, Oak Ridge National Laboratory, Oak Ridge Tennessee 37831, United States
}

Supporting Information

ABSTRACT: The measurement of biological events on the surface of live cells at the single-molecule level is complicated by several factors including high protein densities that are incompatible with single-molecule imaging, cellular autofluorescence, and protein mobility on the cell surface. Here, we fabricated a device composed of an array of nanoscale apertures coupled with a microfluidic delivery system to quantify single-ligand interactions with proteins on the cell surface. We cultured live cells directly on the device and isolated individual epidermal growth factor receptors (EGFRs)

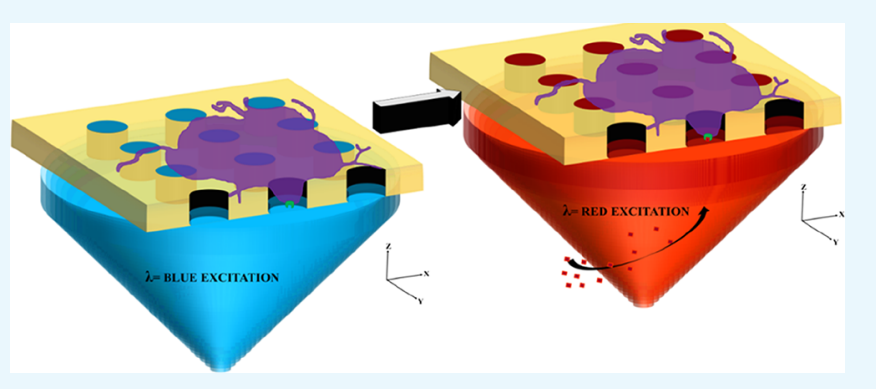
in the apertures while delivering fluorescently labeled epidermal growth factor. We observed single ligands binding to EGFRs, allowing us to quantify the ligand turnover in real time. These results demonstrate that this nanoaperture-coupled microfluidic device allows for the spatial isolation of individual membrane proteins while maintaining them in their cellular environment, providing the capability to monitor single-ligand binding events while maintaining receptors in their physiological environment. These methods should be applicable to a wide range of membrane proteins.

\section{INTRODUCTION}

Cell-surface receptors play a primary role in translating extracellular signals into intracellular messages. ${ }^{1-5}$ A variety of biochemical and microscopy-based techniques have been developed to both monitor ligand interactions with membrane receptors and measure downstream signaling events. ${ }^{1,6-12}$ Whole-cell and single-channel electrophysiology are often employed to monitor the functional activity of ion channels. Fluorescence imaging and complementary techniques provide insights for ligand-receptor binding events, which initiate essential chemical and electrical communication between the intracellular and extracellular environments. ${ }^{4,13-15}$ Understanding these interactions is a key component to deciphering the role these signaling events play in the proliferation of diseases such as cancer. Although existing approaches have added significant understanding of the structure and function of receptors, most involve ensemble measurements that lack the specificity to monitor the dynamics of individual receptorligand activity. ${ }^{16-18}$ Single-molecule studies of membrane proteins are limited by intracellular autofluorescence, weak fluorophore emission, membrane protein mobility on the cell surface, and high protein concentrations that are incompatible with single-molecule measurements. One approach to address the challenge of fluorophore brightness is the use of relatively large but photostable labels such as quantum dots, ${ }^{19}$ which can be utilized to monitor membrane receptor mobility and, in some circumstances, ligand interactions. ${ }^{20,21}$ However, these studies are complicated by the relatively large size of functionalized quantum dots, and their potential toxicity limits their practical use in many applications. ${ }^{22}$

An alternative approach to visualizing single-membrane receptors on the cell surface is the isolation of small extensions of the cell membrane using nanoaperture arrays such as zeromode waveguides (ZMWs), which have previously been used to study single receptors on the surface of live cells. ${ }^{6}$ ZMWs have been utilized for a variety of applications including DNA sequencing ${ }^{23-33}$ and the isolation of purified proteins for single-molecule Förster resonance energy-transfer studies. ZMWs are well-suited for single-molecule studies because the excitation light is confined to a small domain near the entrance of the aperture. Rather than light transmitting through the ZMW, an evanescent wave is generated that exponentially decays away from the entrance and the resulting small spatial dimensions of the focal volume $\left(\sim 10^{-21} \mathrm{~L}\right)$ allow for singlemolecule measurements at micromolar concentrations. ${ }^{25}$ Only fluorescent molecules in the lower portion of the ZMW are

Received: July 5, 2017

Accepted: July 11, 2017

Published: July 25, 2017 
within the observation volume and visible with fluorescence excitation. ZMWs can also be employed for cell-based studies. Proteins at high concentrations on the surface of live cells can be isolated at single-molecule levels in protrusions of small portions of the plasma membrane in the apertures. With the correct properties, metal composition, and size, ZMWs can also be tuned to enhance the fluorescence signal of single fluorophores for both immobilized molecules ${ }^{33-36}$ and those in solution. ${ }^{37-39}$

One major drawback of cell-based studies using standard ZMWs is that the apertures are only accessible from one side of the device. Devices are normally fabricated on a thin $(\sim 150$ $\mu \mathrm{m})$ glass substrate that serves as the backbone for the ZMW while the apertures themselves are patterned in a thin metal film on top of the substrate. This configuration is not compatible with monitoring single-ligand interactions with cell surface receptors, as the glass substrate that serves as a base for the ZMW makes them inaccessible to solution exchange from the side of the device opposite the cells. Recently, the Wanunu group has fabricated a hybrid ZMW with a $5 \mathrm{~nm}$ nanopore drilled through its center. ${ }^{31}$ Fabrication of the device on a silicon nitride membrane allowed solution exchange through the $5 \mathrm{~nm}$ pore and voltage-driven delivery of DNA to the pore for optical and electrical measurements. Arrays of similar nanoporous membranes have also been used as sensors for analyte trapping and monitoring. ${ }^{40-44}$

Here, we developed a new device that integrated ZMW arrays (40000 holes) with microfluidic delivery that was compatible with isolating small portions of the plasma membrane on a live cell. To allow for solution delivery from both sides of the device, we fabricated ZMWs on a $120 \mathrm{~nm}$ thick silicon nitride substrate coated with a $100 \mathrm{~nm}$ thick gold layer and coupled this with the microfluidic device. A network of microfluidic channels was microfabricated in polydimethylsiloxane (PDMS), and one surface of the microfluidic device was then mounted on a glass coverslip while the ZMW was integrated into the opposing surface. The microfluidic cavity between the glass and ZMW was approximately $80 \mu \mathrm{m}$ tall, allowing for fluid to be delivered to the underside of the ZMW device, but still making it compatible with high numerical aperture (NA) objectives necessary for single-molecule imaging. A schematic of the device is shown in Figure 1. This approach provides the benefits achieved with standard ZMWs such as isolating single proteins from high concentrations, limiting cellular autofluorescence, and providing fluorescence enhancement while retaining the freedom to monitor ligand fluorescence delivered via the microfluidic channels. This enabled us to individually address multiple receptors simultaneously and observe ligand binding and unbinding events at each receptor location.

\section{RESULTS AND DISCUSSION}

We demonstrate that our microfluidic-ZMW (mf-ZMW) devices can be used to image single-ligand delivery events by culturing cells that express epidermal growth factor receptors (EGFRs) within the ZMWs. The plasma membrane extended into several of the wells lying under each cell. The receptors are contained in filopodia-like projections into the wells, which limits the number of EGFRs within them. ZMWs are subwavelength apertures, so light is not transmitted through the well. Only receptors near the lower end of the well would be visible. Although the dimensions of the aperture and the extension of filopodia-like structures limit the numbers of

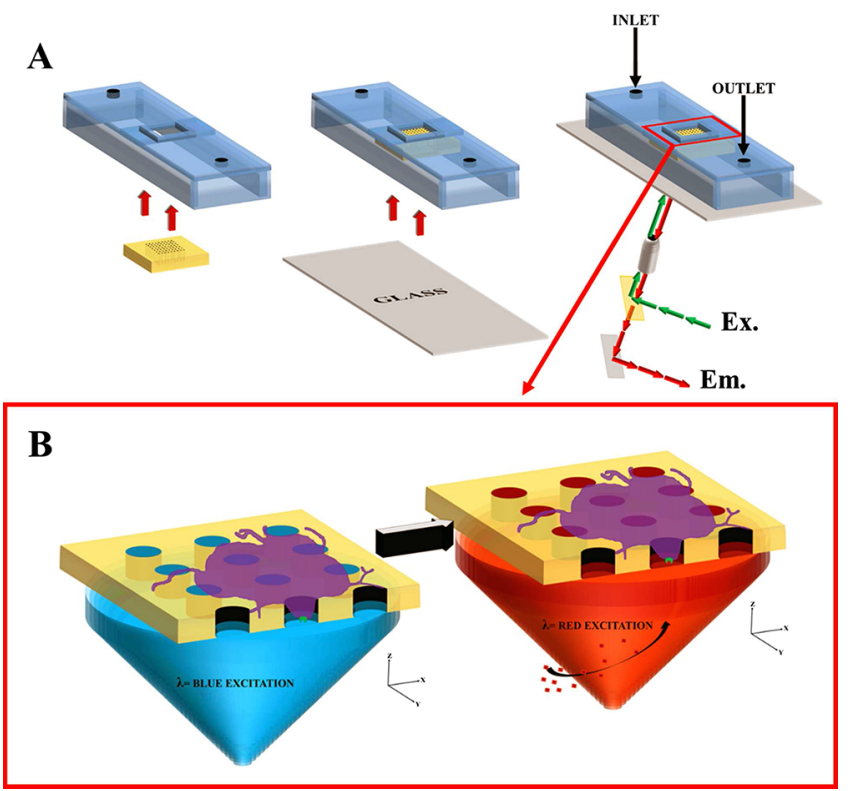

Figure 1. (A) Diagram depicting the assembly of the array and the microfluidic device. The gold-coated $\mathrm{Si}_{x} \mathrm{~N}_{y}$ membrane patterned with $230 \mathrm{~nm}$ diameter ZMWs is bonded to the underside of a PDMS microfluidic delivery system using an oxygen plasma with a glass coverslip capping the bottom. Excitation light is directed through the objective and focused on the sample plane through the microfluidic. Emission is collected by the same objective and directed via a dichroic toward the EMCCD. (B) Schematic showing EGFR-GFP expressing cells integrated with the device and $488 \mathrm{~nm}$ laser excitation being used to visualize receptors in the wells. The cell is shown in purple with an extension of the membrane containing an EGFR shown in green extends into the well. EGF is represented by the red molecule binding to the green receptor in the schematic. A syringe pump is used to wash in EGF-A647, and a $640 \mathrm{~nm}$ laser excitation is used to visualize binding events.

receptors in the wells, there is likely more than a single receptor in the well. However, the dimensions of the apertures limit the number of ligands being delivered from the microfluidic side of the well. At the concentration of ligand used in our studies (20 $\mathrm{nM})$, the simultaneous delivery of more than one ligand to a single well would be a rare event. We delivered fluorescently labeled epidermal growth factor (EGF) via the microfluidic channel (Figure 1). This enabled us to directly observe and quantify binding and unbinding events at the single-ligand level, which consequently allowed us to determine ligand turnover based on single interactions.

Fabrication and Characterization of mf-ZMWs. Gold ZMWs were fabricated on $\sim 120 \mathrm{~nm}$ thick low-stress silicon-rich silicon nitride $\left(\mathrm{Si}_{x} \mathrm{~N}_{y}\right)$ membranes (Figure $\mathrm{S} 1 \mathrm{~B}$ ). This hybrid $\mathrm{ZMW} \mathrm{Si}_{x} \mathrm{~N}_{y}$ membrane acts as a support for the metal film instead of a glass coverslip, which is typically used. Because the $\mathrm{Si}_{x} \mathrm{~N}_{y}$ membrane is $\geq 1400$ times thinner than a glass coverslip, the ZMW features can be patterned through it. The hybrid membrane structure is plasma-bonded with a microfluidic delivery system, allowing cells to adsorb onto the $\mathrm{Si}_{x} \mathrm{~N}_{y}$ side of the ZMW while solution is delivered from a microfluidic channel bonded to the metal side of the membrane. We utilized two different substrates. The first was commercially available porous $\mathrm{Si}_{x} \mathrm{~N}_{y}$ substrates (Norcada Inc.) with $2 \mu \mathrm{m}$ diameter holes. These substrates are compatible with cell culture and integration with a microfluidic device and could also be easily coated with a metal layer; however, the well diameters were too 
large to create the confined focal volume effect present in true ZMWs. However, it was possible to use these larger apertures to optimize the process of integrating the silicon nitride membrane with microfluidics and to verify cell membrane protrusion into the wells and ligand delivery through the microfluidic channels. We coated one side of these membranes with a $10 \mathrm{~nm}$ layer of chromium, followed by a $90 \mathrm{~nm}$ layer of gold. The opposite face of the membrane was bonded to a microfluidic device composed of PDMS (Figure 1). To test the viability of the device, we cultured mouse neuroblastoma (N2a) cells expressing EGFR fused to green fluorescent protein (GFP) directly on the unmodified gold surface of the substrate. Imaging the GFP through the transparent microfluidic cavity showed that the cell plasma membrane had extended into the wells (Figure 2A). We then delivered EGF conjugated to a

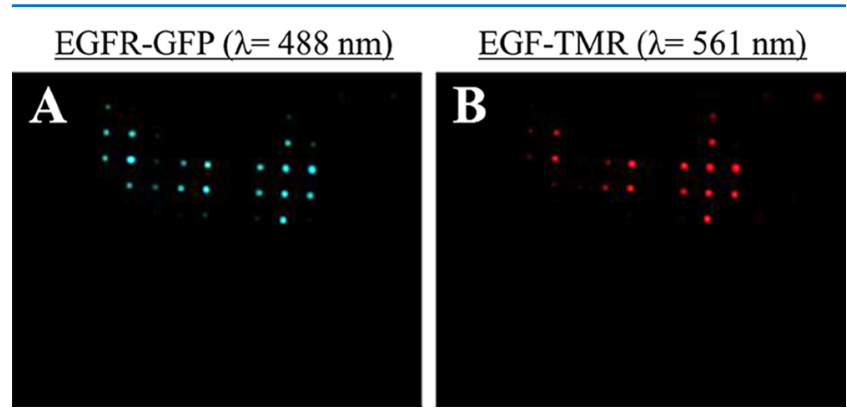

Figure 2. Monitoring EGF-binding events in large apertures. (A) Wide-field fluorescence image ( $488 \mathrm{~nm}$ excitation) from the bottom of a device $(2 \mu \mathrm{m}$ holes $)$ with cells expressing GFP-labeled EGF receptors cultured on top of the device. The plasma membrane containing EGFR-GFP is only visible where it extends into the wells. (B) Wide-field fluorescence image of the same region showing preferential binding of TMR-labeled EGF delivered via the microfluidic channel on the bottom side of the device.

fluorescent label [(tetramethylrhodamine (TMR)] through the microfluidic device. All studies with 2 micron apertures utilized TMR-labeled EGF. Although the cells showed no background fluorescence at $561 \mathrm{~nm}$ excitation prior to delivering EGFTMR, the preferential binding of ligands to EGFR-GFP was apparent postdelivery (Figure 2B). TMR fluorescence was observed only in those wells where GFP fluorescence was also present. These initial results also verified that the microfluidic device could be fabricated thin enough $(<140 \mu \mathrm{m})$ to be compatible with the working distance of high-NA objectives. This demonstration also showed that preferential delivery of a ligand to a cell-surface receptor could be achieved with no apparent nonspecific interactions of the ligand with the substrate or with portions of the cell membrane not containing the EGFR.

We next fabricated devices with $\sim 230 \mathrm{~nm}$ diameter apertures using a substrate composed of $\mathrm{Si}_{x} \mathrm{~N}_{y}$ membranes fabricated at the Center for Nanophase Materials Sciences (CNMS) at Oak Ridge National Laboratory. These apertures are small enough to produce a true ZMW effect. To fabricate these ZMWs, a 120 $\mathrm{nm}$ thick layer of $\mathrm{Si}_{x} \mathrm{~N}_{y}$ was deposited on both sides of a 300 $\mu \mathrm{m}$ thick double-side-polished silicon wafer by using lowpressure chemical vapor deposition (LPCVD). ZMW features were patterned using electron-beam lithography (EBL) on one side of the $\mathrm{Si}_{x} \mathrm{~N}_{y}$ surface (the front side) followed by reactive ion etching (RIE) of the nitride layer down to the silicon substrate (Figure S2). A conventional contact alignment optical lithography process was carried out on the backside of the wafer to pattern a window corresponding to the area, which contained the ZMW features. After etching the $\mathrm{Si}_{x} \mathrm{~N}_{y}$ layer on the backside, the wafer underwent $\mathrm{KOH}$ wet etching to remove the exposed silicon substrate all the way to the nitride membrane on the front side (Figure S2). This resulted in devices composed of a thick silicon frame with a thin silicon nitride membrane suspended across the frame. The ZMWs extended all the way through the $\mathrm{Si}_{x} \mathrm{~N}_{y}$ membrane, allowing access to the holes from either side. This device was then coated with a $5 \mathrm{~nm}$ layer of chrome followed by a $100 \mathrm{~nm}$ layer of gold using thermal evaporation (Figure S2H). The apertures maintained the same diameter after metal deposition (Figure S2G).

A PDMS microfluidic device was fabricated so that the aperture arrays could be integrated into the top internal surface of the microchannels while the bottom surface of the channels was sealed with a $170 \mu \mathrm{m}$ thick glass coverslip (Figure $1 \mathrm{~A}$ and B). The PDMS devices were designed with an internal recessed surface; the ZMW arrays were designed such that the silicon frame surrounding them could be plasma-bonded to the recessed surface inside of the PDMS device. The glass coverslip was then plasma-bonded to the other surface of the PDMS device, creating an enclosed fluidic pathway that could be used to flow fluid past the gold-coated surface of the ZMW arrays, while cells could be cultured on the opposing surface. The apertures of the ZMWs using this fabrication method ranged between 210 and $230 \mathrm{~nm}$, as shown in the scanning electron microscopy image in Figure S2G.

Single-Ligand Binding. We cultured N2a cells expressing EGFR-GFP directly onto the mf-ZMW, and an additional PDMS reservoir was created on the $\mathrm{Si}_{x} \mathrm{~N}_{y}$ side of the membrane to contain the cells and cell media. We were able to monitor the cells using bright-field illumination (Figure 3A) and via GFP
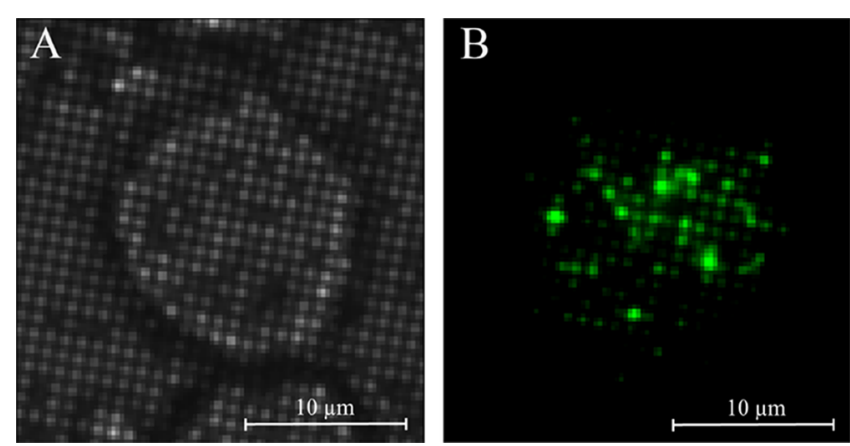

Figure 3. Cell penetration in subwavelength apertures. (A) Bright-field illumination of a mouse neuroblastoma $2 \mathrm{a}(\mathrm{N} 2 \mathrm{a})$ cell expressing EGFR-GFP cultured on a nanoaperture $(\sim 230 \mathrm{~nm})$ device. (B) Same cell under $488 \mathrm{~nm}$ laser excitation from the bottom side of the device showing cell penetration and EGFR-GFP fluorescence in the wells.

fluorescence with $488 \mathrm{~nm}$ excitation (Figure 3B). Excitation light was delivered from the bottom side of the mf-ZMW through the microfluidic channels. High levels of bright-field illumination from the top were sufficient to locate cells with electron-multiplying charge-coupled device (EMCCD) detection. Neither lower level bright field nor laser illumination was sufficient to image through the mostly opaque gold surface. Once the cells were located with bright-field excitation, we used wide-field fluorescence illumination to monitor the emission with an EMCCD to quantify the cell footprint and the number of wells exhibiting plasma membrane protrusions that also 


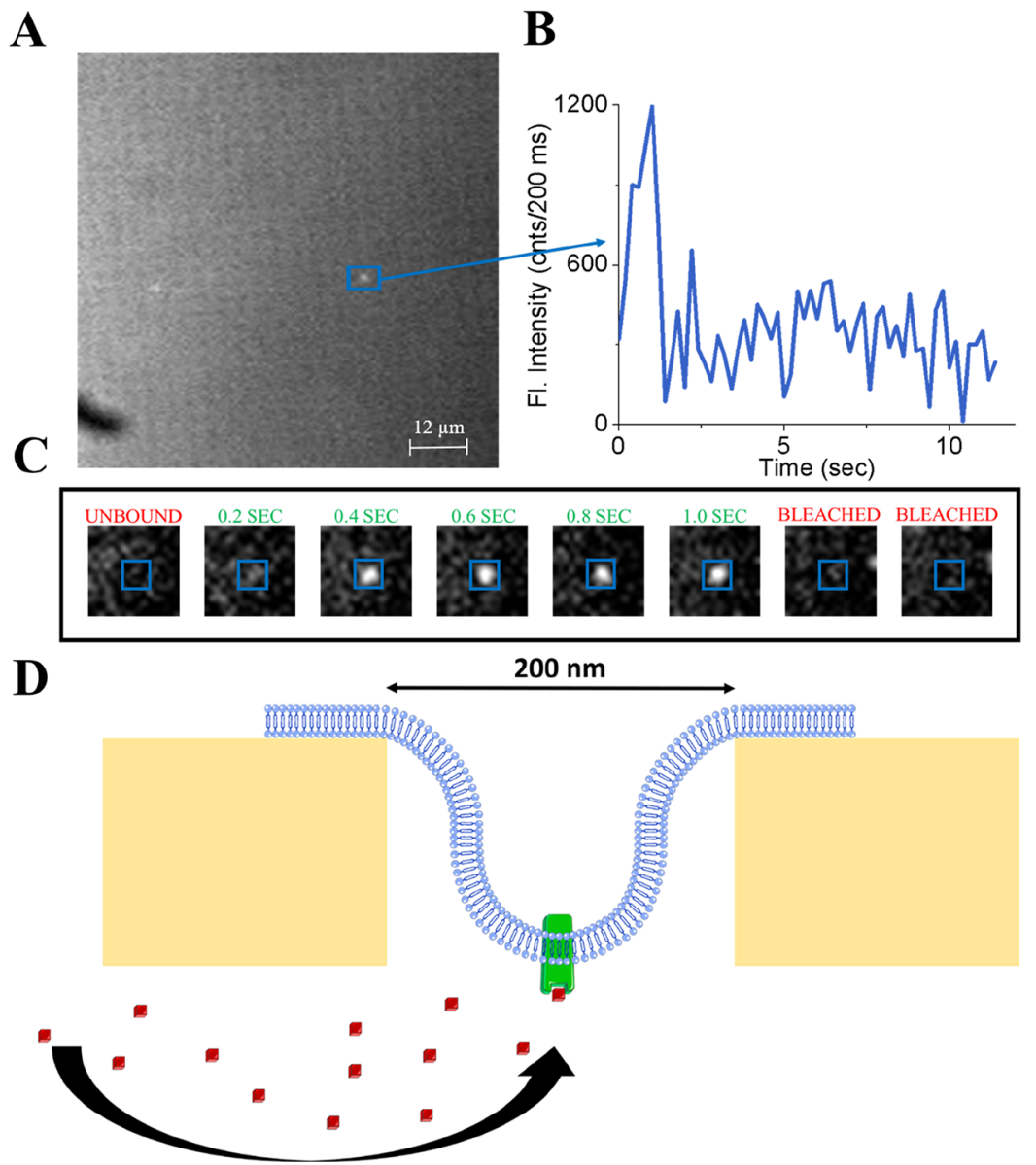

Figure 4. Detecting single-ligand-binding events. (A) EMCCD image of a single-ligand binding to EGFR-GFP in real time. (B) Time trace for the binding event observed in (A) that shows the emitter photobleaching in less than $1 \mathrm{~s}$. (C) Consecutive EMCCD images of the same EGF-A647 showing the sequence from binding to the receptor to photobleaching. (D) Schematic outlining the detection of ligand-receptor interactions, which shows that the EGFR-GFP receptor (green) is incorporated in the plasma membrane lipid bilayer within the gold ZMW, and the EGF-A647 ligands (red) are washed through the microfluidic channel to undergo binding.

contained labeled receptors. Typically, $\sim 20 \%$ of the apertures under a cell footprint exhibited GFP fluorescence, indicating plasma membrane containing EGFRs. Once the apertures containing EGFRs were identified, we delivered a solution containing $20 \mathrm{nM}$ EGF labeled with Alexa 647 (A647) at a constant flow rate through the microfluidic channel to the underside of the ZMW. A647 was used for all single-molecule studies because it is a better single-molecule fluorophore and the plasmon enhancement in gold ZMWs is larger in the red region of the visible spectrum. ${ }^{45}$ While imaging through the microfluidic channel during ligand delivery, diffuse fluorescence was observed and corresponded to EGF-A647 in solution. The presence of this fluorescence signal verified the delivery of EGF to the ZMW, and at this point, we initiated data collection, capturing images at a rate of 5 frames per second. Binding events were observed where EGF molecules became immobilized in the location of EGFR-containing apertures. As shown in Figure 4A,C, a single EGF molecule is readily visible when it binds to an EGFR isolated in an aperture. In areas not containing a bound EGF molecule, only diffuse background fluorescence was observed because of labeled EGF flowing through the device. The single-step photobleaching ${ }^{7,46}$ shown in the representative time trace in Figure $4 \mathrm{~B}$ is indicative of the single-molecule behavior. The time trace shows that while the ligand is bound, the fluorescence intensity is higher for a period of time until the molecule photobleaches or the ligand unbinds. This is also clearly visible in Figure 4C, where the ligand is not present in the first frame just before binding. It is then visible for the next 5 frames and disappears for the final 2 frames after the molecule photobleaches. A schematic illustrating the delivery and binding of single ligands with EGFRs is shown in Figure 4D, demonstrating the use of mf-ZMW devices to monitor ligand-receptor turnover.

Effect of mf-ZMWs on Single-Molecule Emission. Two of the primary challenges in single-molecule imaging are fluorophore brightness and photostability. Plasmonic structures including gold ZMWs have previously been shown to enhance the fluorescence signal of single-molecule fluorophores. ${ }^{35,38,39,47,48}$ To determine whether mf-ZMWs alter the photophysical properties of fluorophores, we isolated single EGF-A647 ligands on the surface of a glass substrate and compared them to the same molecules isolated in mf-ZMWs. To bind EGF-A647 to glass substrates, we functionalized glass coverslips with biotin using $2 \mathrm{mg} / \mathrm{mL}$ Silane-poly(ethylene glycol)-Biotin in 95\% EtOH for $30 \mathrm{~min}$. EGF-A647 was coupled via streptavidin, and the vacant binding site was used to immobilize the molecule on the biotin-coated surface.

We measured the photostability and brightness of fluorophores under both conditions using $640 \mathrm{~nm}$ excitation and EMCCD detection of single molecules. Fluorophores on a 


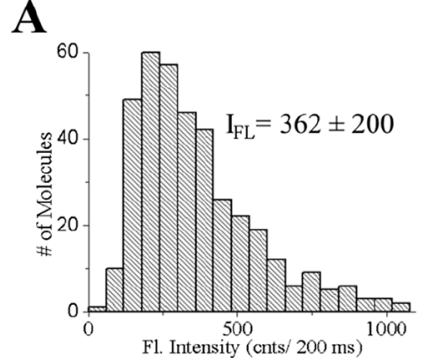

D

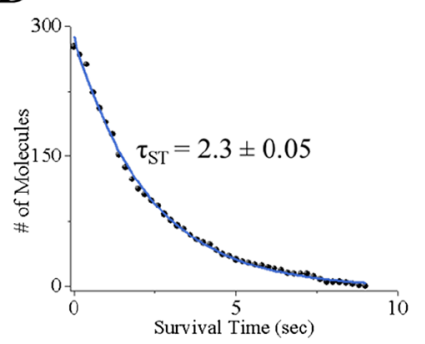

B

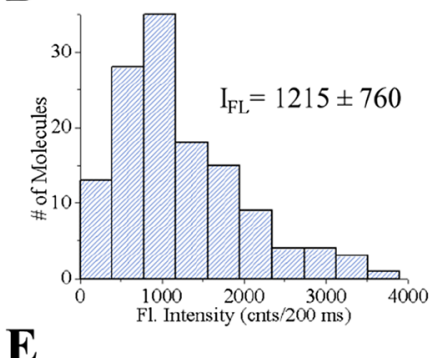

$\mathbf{E}$

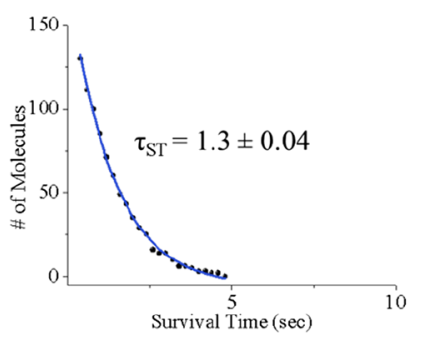

C

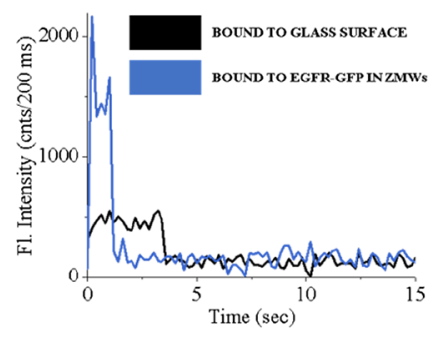

$\mathbf{F}$

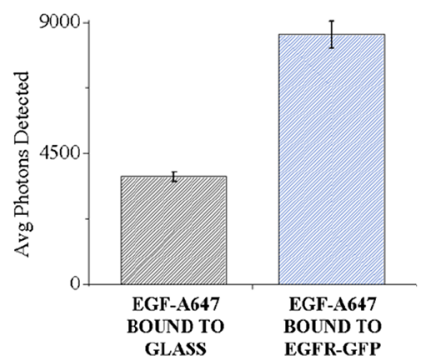

Figure 5. Photophysical characterization of A647 in mf-ZMWs. (A) Histogram of the fluorescence intensity of single molecules isolated on a glass surface. (B) Histogram of the fluorescence intensity of single molecules isolated via binding of EGF to EGFR in nanoscale apertures. (C) Representative time traces of EGF-A647 bound to glass (black) and EGF-A647 bound to EGFR-GFP in gold ZMWs (blue) showing that molecules in ZMWs are brighter. (D) Plot of the average survival time $\left(\tau_{\mathrm{ST}}\right)$ for single EGF-A647 molecules on a glass surface showing that they survive for approximately $2.3 \mathrm{~s}$. (E) Plot of the average survival time $\left(\tau_{\mathrm{ST}}\right)$ for single EGF-A647 molecules bound to EGFR in the nanoarray showing that they survive for approximately $1.3 \mathrm{~s}$. (F) Plot comparing the average number of detected photons $\left(I_{\mathrm{FL}} \times \tau_{\mathrm{ST}}\right)$ for single molecules on glass to those in mfZMWs showing roughly a 2 -fold increase. Error bars represent the standard deviation.

glass substrate exhibited a fluorescence intensity $\left(I_{\mathrm{Fl}}\right)$ of 362 counts per $200 \mathrm{~ms}$ (Figure 5A), whereas molecules in the mfZMW showed an $I_{\mathrm{Fl}}$ of 1215 counts per $200 \mathrm{~ms}$ (Figure $5 \mathrm{~B}$ ). These studies indicate that similar to standard ZMWs, mfZMWs enhance the fluorescence signal of nearby fluorophores. Because the photostability of molecules will directly impact the duration that ligands can be monitored after delivery to the cell, we also compared the photostability of EGF-A647 on glass substrates with that in the mf-ZMWs. Molecules on a glass substrate had a survival time of $2.3 \mathrm{~s}$ (Figure 5D) and those in mf-ZMWs had a survival time of $1.3 \mathrm{~s}$. The increase in fluorescence intensity and the decrease in photostability are similar to what has been observed for molecules isolated in standard gold ZMWs. ${ }^{35}$ Representative time traces of molecules on glass versus that in an mf-ZMW are shown in Figure 5C. We then calculated the relative number of detected photons for single EGF-A647 ligands using the emission intensity and photostability of individual molecules. Molecules in mf-ZMWs yielded $8580 \pm 464$ counts versus an average of only $3691 \pm$ 163 counts when isolated on the glass surface (Figure 5F). This shows a greater than 2-fold increase in the number of detected photons for the ligand bound to the EGFR as seen in the representative time traces (Figure $5 \mathrm{C}$ ). The increase in the number of detected photons in the presence of the $\mathrm{mf}-\mathrm{ZMW}$ is likely due to a combination of plasmon-related enhancement occurring during both excitation and emission and arising from the interaction of light with the metal nanostructures. Gold ZMWs in particular have recently been observed to contribute to a similar fluorescence enhancement of fluorophores, which excite at $640 \mathrm{~nm}$ excitation. ${ }^{35}$

Time-Lapse Single-Molecule Imaging to Determine Ligand Turnover. Fluorophore survival times before photobleaching limit the duration that we can continuously monitor ligand-receptor interactions. Constant excitation leads to the detection of ligands for approximately $4-6$ frames $(0.8-1.2 \mathrm{~s}$ of continuous imaging) before photobleaching. This is likely shorter than the lifetime of EGFRs on the plasma membrane. Common strategies to increase photostability such as reducing the excitation intensity and the use of an oxygen scavenging system were not compatible with the mf-ZMW device. Our excitation intensity was set at the minimum level to be able to distinguish the fluorophore signal from the background. Strategies to eliminate oxygen, such as the use of oxygen scavenging systems in sealed environments, ${ }^{49,50}$ can alter the physiological environment and are often not compatible with live cell studies. Additionally, the live cell chamber on the top of the device combined with the fluid exchange of the mf-ZMW substrate made it impossible to isolate fluorophores from the environment as would be necessary for oxygen scavenging systems. To increase the longevity of our observation window, we utilized time-lapse interval-based single-molecule detection. This allowed us to extend the total amount of time a ligand is visible by shortening the time the molecule was continuously exposed to laser excitation. While the number of frames (4-6) remained the same, increasing the interval between frames allowed us to extend the observation times long enough to encompass the turnover of EGF-A647. For example, using a time lapse with the laser shuttered for $5 \mathrm{~s}$ between frames extends the experimental window for ligand observation to $21 \mathrm{~s}$. We performed time-lapse experiments at increasing intervals until the average observed time of the bound ligand decreased below the number of frames needed to photobleach the molecule. Time-lapse interval measurements on the binding of EGF-EGFR were performed by using the same experimental conditions as with the continuous measurements, but with 1,2 , $5,10,25,40$, and $100 \mathrm{~s}$ shuttered intervals in between frames. The longer the time interval, the longer the A647 fluorophore on EGF can be monitored for continued binding to the EGFR. We observed an average of 3-5 frames per EGF-EGFR binding event for all time-lapse interval measurements up 


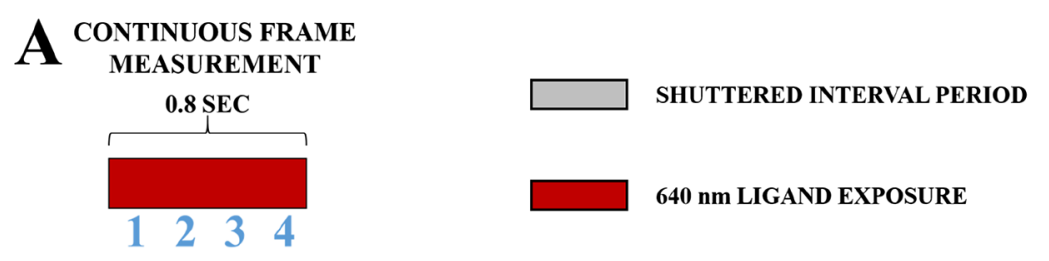

\section{SECOND TIME LAPSE INTERVAL MEASUREMENT}
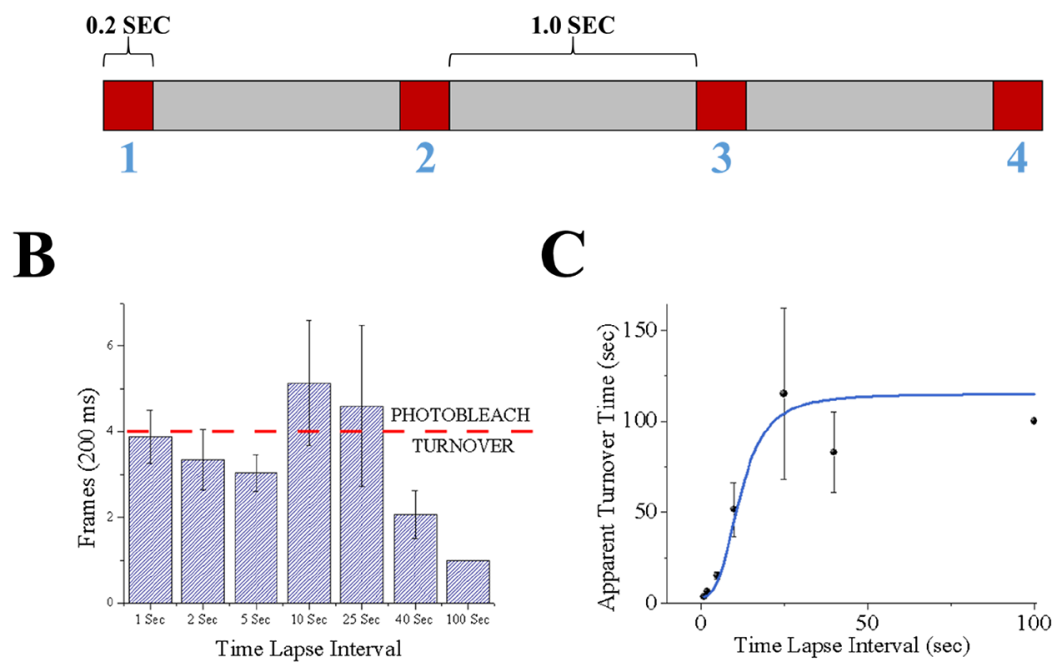

Figure 6. Turnover of EGFR and EGF binding. (A) Schematic comparing continuous excitation to time-lapse measurements. In each case, a total of 4 frames are taken, but with a built-in shuttered time interval, and the total duration of the experiments is extended because of the interval. Fluorophores that bleach in approximately 4 frames can be used to measure dynamics on a longer time scale by introducing a shuttered interval between frames. (B) Bar graph showing the average number of frames a fluorophore is visible during a series of interval studies. For $1-25 \mathrm{~s}$ intervals, there is no significant difference between the groups $(t$-test, $p<0.01)$. The average number of frames is equal to or greater than the 4 frame bleaching level (dotted red line), indicating that the molecules are bleaching before turnover. Time-lapse intervals ranging from 40 to $100 \mathrm{~s}$ show a drop below the 4 frame bleaching level, indicating that the ligand is departing the surface prior to photobleaching. (C) Plot of interval time vs the average time molecules were observable. At longer time-lapse intervals, a clear plateau is reached, showing that the turnover time is between 80 and $100 \mathrm{~s}$.

through intervals of $25 \mathrm{~s}$. At 40 and $100 \mathrm{~s}$ intervals, there is a marked decrease in the number of frames for which a single ligand is visible on the mf-ZMW surface (Figure 6B). On the basis of our characterization of the photostability of A647 (4-6 frames on average), a decrease in the number of frames observed below this threshold can be attributed to the ligand unbinding from the receptor and diffusing out of the ZMW aperture prior to being photobleached. On average, the ligand was visibly bound to the receptor for 2 frames at $40 \mathrm{~s}$ intervals and 1 frame with $100 \mathrm{~s}$ intervals (Figure 6B).

We also plotted the time-lapse interval versus the average duration of molecules that were observed for each interval. The observed duration was defined as the number of frames multiplied by the frame interval. For example, with a $1 \mathrm{~s}$ interval, the average number of frames ( $200 \mathrm{~ms}$ exposure) was $\sim 4$ frames and the duration was calculated as $3.8 \mathrm{~s}$ (Figure 6A). A clear plateau was seen in the length of time the molecules were observed as the time-lapse interval increases (Figure 6C). When time intervals are not long enough to extend the observation window beyond the turnover time, the observation time increases sharply between consecutive intervals as seen in intervals of $1-25 \mathrm{~s}$ (Figure 6B). The plateau observed at the 40 $\mathrm{s}$ interval indicates that departure occurs prior to reaching the 4-6 frame photobleaching regime. We attribute this to singleEGF turnover when bound to EGFRs. Although ligand-induced endocytosis of the EGFR typically occurs on a longer time frame $^{51}$ than the intervals studied here, our experiment does not differentiate between departure of the A647-labeled EGF due to unbinding and the loss of fluorescence due to internalization of the receptor-ligand complex. On the basis of the differences in time scales for unbinding and internalization, the vast majority of our observed events are likely due to ligand turnover. These studies shown here are the first to observe single-ligand turnover on the surface of a live cell and produce rates similar to those shown in the studies of isolated EGF and EGFR on a glass substrate. ${ }^{52}$

\section{CONCLUSIONS}

We have developed a hybrid system composed of arrays of nanoapertures on a $\mathrm{Si}_{x} \mathrm{~N}_{y}$ membrane integrated with a microfluidic delivery system. These devices offer many of the same benefits as standard ZMWs such as fluorescence enhancement and single-molecule isolation. However, they also offer the additional advantage of being compatible with isolating nanoscale domains of the plasma membrane, which can then be accessed using the microfluidic device. The spatial isolation of these small domains provides a way to monitor single proteins on the surface of a live cell. Using EGFR and EGF, we have shown that mf-ZMWs can be used to monitor single-ligand binding to EGF receptors. By reducing the ZMW diameter to $\sim 200 \mathrm{~nm}$, this method has the potential to be a powerful tool capable of monitoring interactions between 
ligands and virtually any receptor, by enabling the spatial isolation of single receptors on the surface of a cell and providing access to these receptors via microfluidic channels. The ability to isolate several molecules in parallel on a cell surface and individually monitor receptor activity should have a wide range of applications including functional measurements of single channels, ligand-receptor interactions, and receptor turnover.

\section{EXPERIMENTAL SECTION}

Fabrication of $230 \mathrm{~nm}$ ZMWs in Silicon Nitride Membranes. All $\mathrm{Si}_{x} \mathrm{~N}_{y}$ membrane fabrication processes were conducted at the CNMS at Oak Ridge National Laboratory. Fabrication was performed using EBL to pattern the ZMW features onto one side of a $\mathrm{Si}_{x} \mathrm{~N}_{y}$-coated silicon wafer (front side), whereas photolithography was used to pattern larger (microscale) features onto the backside. A combination of RIE and wet chemical etching was used to produce nanoporous $\mathrm{Si}_{x} \mathrm{~N}_{y}$ membranes suspended on a silicon substrate. Prior to beginning the fabrication procedure, low-stress silicon-rich silicon nitride (120 nm thickness) was deposited onto both sides of $300 \mu \mathrm{m}$ thick 4 in. double-side polished silicon wafers by the LPCVD process (Figure S2A).

ZMW features were defined using a JEOL JBX-9300FS EBL system. First, the nitride-coated silicon wafers were spin-coated with a ZEP-520A electron beam resist (Zeon Chemicals, Japan) at $2000 \mathrm{rpm}$ for $45 \mathrm{~s}$ and baked on a hot plate at $180{ }^{\circ} \mathrm{C}$ for 2 min (Figure S2B). The wafers were then exposed at a dose of $400 \mu \mathrm{C} / \mathrm{cm}^{2}$ with $100 \mathrm{kV}$ acceleration voltage and $2 \mathrm{nA}$ beam current. The patterned wafers were then developed for $30 \mathrm{~s}$ in a xylenes solution, rinsed with isopropanol (IPA), and dried with $\mathrm{N}_{2}$ gas (Figure $\mathrm{S} 2 \mathrm{C}$ ), followed by a $6 \mathrm{~s}$ oxygen plasma exposure at $100 \mathrm{~W}$ and $10 \mathrm{sccm} \mathrm{O}$. The patterned silicon nitride layers were then etched in an inductively coupled ion plasma etching system (Oxford Plasmalab 100) down to the silicon substrate with the e-beam resist as the etch mask (Figure S2D). The process was carried out in a mixture of $45 \mathrm{sccm}$ octofluorocyclobutane $\left(\mathrm{C}_{4} \mathrm{~F}_{8}\right)$ and $2 \mathrm{sccm} \mathrm{O}_{2}$ gases at $200 \mathrm{~W}$ radio frequency (rf) and $15{ }^{\circ} \mathrm{C}$. The electron beam resist was then removed by soaking the wafers in acetone for $60 \mathrm{~min}$ followed by an oxygen plasma exposure to $100 \mathrm{sccm} \mathrm{O}_{2}$ at 10 $\mathrm{W}$ rf for $5 \mathrm{~min}$ in the plasma etching system to remove any organic residue (Figure S2E).

Once the ZMW arrays were patterned onto the top side of the $\mathrm{Si}_{x} \mathrm{~N}_{y}$-coated wafers, the backside of the wafers was patterned using conventional contact alignment optical lithography and etched to complete the nanoporous membranes (Figure S2F). Prior to photoresist coating, an adhesion promoter MCC Primer 80/20 (MicroChem, Westborough, MA) was spin-coated onto the $\mathrm{Si}_{x} \mathrm{~N}_{y}$ surface at $3000 \mathrm{rpm}$ for 45 s. A positive photoresist MICROPOSIT S1818 (MicroChem, Westborough, MA) was used to pattern the backside silicon nitride window. The wafers were then baked on a hot plate at $115^{\circ} \mathrm{C}$ for $1 \mathrm{~min}$ and exposed for $5 \mathrm{~s}$ using a Quintel contact aligner. The wafers were developed using CD-26 solution $(<5 \%$ tetramethylammonium hydroxide, MicroChem, Westborough, MA) for $1 \mathrm{~min}$ and $50 \mathrm{~s}$, rinsed with deionized (DI) $\mathrm{H}_{2} \mathrm{O}$, and dried with $\mathrm{N}_{2}$ gas. The wafers were exposed to an oxygen plasma for $1 \mathrm{~min}$ to remove any residual photoresist. The backside silicon nitride window was etched using the same recipe as for the front side nitride. A 5 min oxygen plasma exposure was used to remove the remaining S1818 photoresist.
The final step in the process is to chemically etch the $300 \mu \mathrm{m}$ thick silicon from the backside of the wafers, to create suspended nanoporous silicon nitride membranes. The wafers were submerged in a $30 \%$ potassium hydroxide $(\mathrm{KOH})$ bath at $80{ }^{\circ} \mathrm{C}$ for a period of $3 \mathrm{~h} .{ }^{53}$ An apparatus was used to protect the front side of the wafer, leaving the silicon nitride membrane unharmed (Figure S2F). Upon removing from the $\mathrm{KOH}$ solution, the wafers were rinsed gently with $\mathrm{DI} \mathrm{H}_{2} \mathrm{O}$ and IPA. The wafers were placed in an electron beam evaporator where 5 $\mathrm{nm}$ of chromium and $100 \mathrm{~nm}$ of gold were deposited onto the front side of the wafer (Figure S2H). The wafers were then cleaved into separate membrane devices. With the deposition process completed, the perforated edges were separated from one another and the individual membranes were ready for integration into microfluidic devices (Figure $\mathrm{S} 2 \mathrm{H}$ ).

Cell Culture on Silicon Nitride Membranes. Mouse neuroblastoma cells were transfected with EGFR labeled with GFP. The cells ( 3 million) were plated in a T-75 flask in N2aspecific media (45\% Opti-MEM, 45\% Dulbecco's modified Eagle's medium, and $10 \%$ fetal bovine serum) and incubated for $24 \mathrm{~h}$. The cells were transfected on day 2 using a modification of the standard Lipofectamine 2000 protocol. EGFR-GFP (3500 ng) was added to a $1.5 \mathrm{~mL}$ microcentrifuge tube containing $250 \mu \mathrm{L}$ of Opti-MEM. Separately, $14 \mu \mathrm{L}$ of Lipofectamine $2 \mathrm{k}$ was added to a second microcentrifuge tube containing $250 \mu \mathrm{L}$ of Opti-MEM. Both tubes were allowed to equilibrate for $5 \mathrm{~min}$. During this period of time, the N2a media were removed from the flask and replaced with $10 \mathrm{~mL}$ of Opti-MEM. When the $5 \mathrm{~min}$ waiting period was complete, the two microcentrifuge tubes were mixed together and left to incubate for an additional $25 \mathrm{~min}$. At the end of the $25 \mathrm{~min}$ waiting period, the solution in the microcentrifuge tube was added to the T-75 flask and placed back in the incubator for a period of $24 \mathrm{~h}$. After $24 \mathrm{~h}$, the transfection mix was removed from the flask and the cells were rinsed with phosphatebuffered saline (PBS). After rinsing, $5 \mathrm{~mL}$ of Versene was added to the flask for $5 \mathrm{~min}$ to resuspend the cells into the solution. The cell solution was then added to a conical tube and centrifuged at $1000 \mathrm{rpm}$ for $5 \mathrm{~min}$ to pellet the cells. The Versene solution was removed from the conical tube, and $2 \mathrm{~mL}$ of fresh N2a media was added to the pellet. The cell concentration was measured using a cytometer, and the concentration was adjusted to attain a concentration of 100 cells $/ \mu \mathrm{L}$. The cell suspension was then added to the membrane surface of the mf-ZMW devices. The devices were placed in the incubator for $30 \mathrm{~min}$ to allow the cells to settle onto the membranes; after this time, $400 \mu \mathrm{L}$ of $\mathrm{N} 2 \mathrm{a}$ media was added to the surface and the devices were returned to the incubator until imaging the following day.

Microscale Aperture Studies. Silicon nitride membranes with $2 \mu \mathrm{m}$ apertures were purchased from Norcada Inc. to be used in the microfluidic devices. These membranes were coated with $10 \mathrm{~nm}$ of chromium followed by $90 \mathrm{~nm}$ of gold using sputter deposition. The coated membranes were then plasmabonded to the top inner surface of PDMS microfluidic devices using a Harrick PDC-32G plasma cleaner; to accomplish this, the membranes and PDMS were exposed to an oxygen plasma for $30 \mathrm{~s}$ and then quickly removed from the vacuum chamber, and the two surfaces to be bonded were pressed together to make a permanent seal (Figure 1A). This bonding process was then repeated to plasma-bond the bottom surface of the PDMS device to a clean, $150 \mu \mathrm{m}$ thick glass coverslip (Figure 1B). 
Transfected N2a cells were added to the devices as described in the previous section and were imaged with an Olympus $60 \times$ water objective and wide-field epifluorescence laser excitation. Before moving forward with the microfluidic delivery portion of the experiment, $488 \mathrm{~nm}$ laser excitation was used to confirm the existence of EGFR-GFP-transfected cells with membrane protrusions in the ZMWs. Next, the system was observed at $561 \mathrm{~nm}$ excitation to ensure that there was no background fluorescence to compete with the ligand EGF-TMR. Bovine serum albumin (BSA), which is known to adsorb onto surfaces and prevent nonspecific binding, ${ }^{54}$ was diluted to $0.1 \%$ in PBS $1 \times$ and flushed through the microfluidic channels for $10 \mathrm{~min}$ using a syringe pump. Next, $10 \mathrm{nM}$ EGF-TMR (in $0.01 \%$ BSA) was flushed through the microfluidic channels for a period of $4 \mathrm{~min}$. BSA rinsate $(0.1 \%$ in PBS $1 \times)$ was washed through the channels once more to remove any unbound EGF-TMR conjugates. Images of the devices were captured with a $200 \mathrm{~ms}$ exposure time per frame on an inverted microscope to show the clear presence of EGFR-GFP with $488 \mathrm{~nm}$ excitation and the specific binding of the ligand EGFTMR in the same locations using $561 \mathrm{~nm}$ excitation.

Single-Ligand Binding. Gold-coated $\mathrm{Si}_{x} \mathrm{~N}_{y}$ membrane ZMWs with $\sim 230 \mathrm{~nm}$ diameter apertures were plasma-bonded to the inside of the microfluidic delivery system, which were then plasma-bonded to a clean $22 \times 22 \mathrm{~mm} \# 1.5$ glass coverslip. The ZMW was oriented such that the gold-coated surface was facing down toward the glass coverslip and the $\mathrm{Si}_{x} \mathrm{~N}_{y}$ side was facing up. The distance between the gold-coated surface and the top of the glass coverslip was $<100 \mu \mathrm{m}$, making it possible to use high NA objectives. N2a cells expressing EGFR-GFP were then plated on the $\mathrm{Si}_{x} \mathrm{~N}_{y}$ surface of the mfZMW devices.

Imaging of the mf-ZMW devices was performed on an inverted microscope (Olympus IX 81) using a 1.49 NA 100X oil immersion objective. An Andor iXon I3 EMCCD camera was used to collect all data during these steps. First, the membrane was brought into focus using bright-field illumination; because of the semitransparency of $100 \mathrm{~nm}$ thick gold, the N2a cells could be located during this step, despite imaging through the ZMW surface. The regions with cells were then exposed to $488 \mathrm{~nm}$ excitation (Cobolt MLD) to excite the GFP on the plasma membrane surface and determine the presence of GFP-labeled receptors in the wells. Once the cells were located, a Cole-Parmer syringe pump was used to deliver a buffer solution containing $0.1 \%$ BSA in PBS $1 \times$ through the channel for a period of $10 \mathrm{~min}$ at a flow rate of $20 \mu \mathrm{L} / \mathrm{min}$. The position of the cells was noted on the camera by capturing a $200 \mathrm{~ms}$ snapshot with $488 \mathrm{~nm}$ excitation, and a second snapshot $(200 \mathrm{~ms})$ was captured using $640 \mathrm{~nm}$ excitation (Cobolt MLD) to determine the fluorescence background signal in the absence of A647.

A new solution containing $20 \mathrm{nM}$ EGF-A647 in $0.1 \%$ BSA in PBS $1 \times$ was delivered to the mf-ZMW devices at a flow rate of $10 \mu \mathrm{L} / \mathrm{min}$ while monitoring the ZMW surface in real time with $640 \mathrm{~nm}$ excitation. At higher concentrations of fluorescently labeled EGF, background fluorescence from diffusing EGF obscured the signal from those bound to the EGFR on the cell membrane. As soon as the fluorophores begin passing into the field of view, the flow rate was reduced to 1 $\mu \mathrm{L} / \mathrm{min}$ for the data collection process. The slower flow rate was used to avoid solution turbulence that caused bubbling and disrupted single-molecule data collection. Data were collected by using an electronic shuttering system to accurately control the timing of exposures to the $640 \mathrm{~nm}$ laser. Continuous movies were collected without pause in between frames, as well as time-lapse interval movies were collected with pauses between frames that included $1,2,5,10,25,40$, and $100 \mathrm{~s}$ intervals.

Data Analysis. All images were analyzed in ImageJ (NIH), and data analysis was performed using OriginPro. Regions of interest (ROIs) for single-ligand binding events were selected by using the Time Series Analyzer 3.0 plugin with a square shape and $4 \times 4$ pixel size. These ROIs were overlaid with 488 $\mathrm{nm}$ excitation snapshots of a cell to ensure that an event occurred within a well on the plasma membrane. The ROIs were then measured for their longevity (fluorophore survival time) and brightness (fluorescence intensity), which was the average fluorescence intensity minus the fluorescence background after photobleaching. These values were then averaged, and the error bars represent the standard deviation of this population. For time-lapse studies, the number of frames each single molecule lasted was averaged for each of the intervals and the error was calculated as the standard deviation of the same population. Any fluorophores that were present during the first frame of the time-lapse interval measurements were not included in the final data, as it was impossible to determine how long the fluorophore was present before the movie began.

\section{ASSOCIATED CONTENT}

\section{Supporting Information}

The Supporting Information is available free of charge on the ACS Publications website at DOI: 10.1021/acsomega.7b00934.

Schematic comparing standard ZMW and mf-ZMW, fabrication schematic of mf-ZMWs, and control image of binding specificity (PDF)

\section{AUTHOR INFORMATION}

\section{Corresponding Authors}

*E-mail: c.trinkle@uky.edu (C.A.T.).

*E-mail: chris.richards@uky.edu (C.I.R.).

ORCID

Christopher I. Richards: 0000-0003-0019-1989

Author Contributions

"W.E.M. and N.G. contributed equally.

\section{Author Contributions}

W.E.M., C.I.R., C.A.T., and C.P.C. planned experiments. W.E.M., B.R.S., E.M., and N.G. performed experiments. All authors have given approval to the final version of the manuscript.

\section{Notes}

The authors declare no competing financial interest.

\section{ACKNOWLEDGMENTS}

The authors acknowledge A. Sorkin (Professor, Department of Pharmacology, University of Colorado) for EGFR-GFP plasmid through Addgene (plasmid 32751). This work was funded through the Human Frontiers Science Program (RGY0081/2014) and the National Institute of Health DA 038817.

\section{REFERENCES}

(1) Fox, A. M.; Moonschi, F. H.; Richards, C. I. The nicotine metabolite, cotinine, alters the assembly and trafficking of a subset of 
nicotinic acetylcholine receptors. J. Biol. Chem. 2015, 290, 2440324412.

(2) Rajan, S. S.; Liu, H. Y.; Vu, T. Q. Ligand-bound quantum dot probes for studying the molecular scale dynamics of receptor endocytic trafficking in live cells. ACS Nano 2008, 2, 1153-1166.

(3) Carter, R. E.; Sorkin, A. Endocytosis of functional epidermal growth factor receptor-green fluorescent protein chimera. J. Biol. Chem. 1998, 273, 35000-35007.

(4) Crites, T. J.; Chen, L.; Varma, R. A TIRF microscopy technique for real-time, simultaneous imaging of the TCR and its associated signaling proteins. J. Visualized Exp. 2012, 61, No. e3892.

(5) Normanno, N.; De Luca, A.; Bianco, C.; Strizzi, L.; Mancino, M.; Maiello, M. R.; Carotenuto, A.; De Feo, G.; Caponigro, F.; Salomon, D. S. Epidermal growth factor receptor (EGFR) signaling in cancer. Gene 2006, 366, 2-16.

(6) Richards, C. I.; Luong, K.; Srinivasan, R.; Turner, S. W.; Dougherty, D. A.; Korlach, J.; Lester, H. A. Live-Cell Imaging of Single Receptor Composition Using Zero-Mode Waveguide Nanostructures. Nano Lett. 2012, 12, 3690-3694.

(7) Moonschi, F. H.; Effinger, A. K.; Zhang, X.; Martin, W. E.; Fox, A. M.; Heidary, D. K.; DeRouchey, J. E.; Richards, C. I. Cell-derived vesicles for single-molecule imaging of membrane proteins. Angew. Chem. 2015, 54, 481-484.

(8) Liu, H.-W.; Cosa, G.; Landes, C. F.; Zeng, Y.; Kovaleski, B. J.; Mullen, D. G.; Barany, G.; Musier-Forsyth, K.; Barbara, P. F. Singlemolecule FRET studies of important intermediates in the nucleocapsid-protein-chaperoned minus-strand transfer step in HIV1 reverse transcription. Biophys. J. 2005, 89, 3470-3479.

(9) König, I.; Zarrine-Afsar, A.; Aznauryan, M.; Soranno, A.; Wunderlich, B.; Dingfelder, F.; Stüber, J. C.; Plückthun, A.; Nettels, D.; Schuler, B. Single-molecule spectroscopy of protein conformational dynamics in live eukaryotic cells. Nat. Methods 2015, 12, 773779.

(10) Yanagida, T.; Sako, Y.; Minoghchi, S. Single-molecule imaging of EGFR signalling on the surface of living cells. Nat. Cell Biol. 2000, 2, $168-172$.

(11) Sako, Y.; Uyemura, T. Total internal reflection fluorescence microscopy for single-molecule imaging in living cells. Cell Struct. Funct. 2002, 27, 357-365.

(12) Riven, I.; Kalmanzon, E.; Segev, L.; Reuveny, E. Conformational rearrangements associated with the gating of the $G$ protein-coupled potassium channel revealed by FRET microscopy. Neuron 2003, 38, 225-235.

(13) Ianoul, A.; Street, M.; Grant, D.; Pezacki, J.; Taylor, R. S.; Johnston, L. J. Near-field scanning fluorescence microscopy study of ion channel clusters in cardiac myocyte membranes. Biophys. J. 2004, $87,3525-3535$.

(14) Demuro, A.; Parker, I. Imaging single-channel calcium microdomains. Cell Calcium 2006, 40, 413-422.

(15) Parker, I.; Smith, I. F. Recording single-channel activity of inositol trisphosphate receptors in intact cells with a microscope, not a patch clamp. J. Gen. Physiol. 2010, 136, 119-127.

(16) Ferguson, K. M.; Berger, M. B.; Mendrola, J. M.; Cho, H.-S.; Leahy, D. J.; Lemmon, M. A. EGF activates its receptor by removing interactions that autoinhibit ectodomain dimerization. Mol. Cell 2003, 11, 507-517.

(17) Bouyain, S.; Longo, P. A.; Li, S.; Ferguson, K. M.; Leahy, D. J. The extracellular region of ErbB4 adopts a tethered conformation in the absence of ligand. Proc. Natl. Acad. Sci. U.S.A. 2005, 102, 1502415029.

(18) Dawson, J. P.; Berger, M. B.; Lin, C.-C.; Schlessinger, J.; Lemmon, M. A.; Ferguson, K. M. Epidermal growth factor receptor dimerization and activation require ligand-induced conformational changes in the dimer interface. Mol. Cell. Biol. 2005, 25, 7734-7742.

(19) Ohyanagi, T.; Shima, T.; Okada, Y.; Tsukasaki, Y.; Komatsuzaki, A.; Tsuboi, S.; Jin, T. Compact and stable SNAP ligand-conjugated quantum dots as a fluorescent probe for single-molecule imaging of dynein motor protein. Chem. Commun. 2015, 51, 14836-14839.
(20) Huang, T.; Xu, X.-H. N. Multicolored nanometre-resolution mapping of single protein-ligand binding complexes using far-field photostable optical nanoscopy (PHOTON). Nanoscale 2011, 3, $3567-3572$.

(21) Huang, T.; Browning, L. M.; Xu, X.-H. N. Far-field photostable optical nanoscopy (PHOTON) for real-time super-resolution singlemolecular imaging of signaling pathways of single live cells. Nanoscale 2012, 4, 2797-2812.

(22) Lim, Y. T.; Kim, S.; Nakayama, A.; Stott, N. E.; Bawendi, M. G.; Frangioni, J. V. Selection of quantum dot wavelengths for biomedical assays and imaging. Mol. Imaging 2003, 2, 50-64.

(23) Korlach, J.; Marks, P. J.; Cicero, R. L.; Gray, J. J.; Murphy, D. L.; Roitman, D. B.; Pham, T. T.; Otto, G. A.; Foquet, M.; Turner, S. W. Selective aluminum passivation for targeted immobilization of single DNA polymerase molecules in zero-mode waveguide nanostructures. Proc. Natl. Acad. Sci. U.S.A. 2008, 105, 1176-1181.

(24) Moran-Mirabal, J. M.; Torres, A. J.; Samiee, K. T.; Baird, B. A.; Craighead, H. G. Cell Investigation of Nanostructures: Zero-Mode Waveguides for Plasma Membrane Studies with Single Molecule Resolution. Nanotechnology 2007, 18, 195101.

(25) Levene, M. J.; Korlach, J.; Turner, S. W.; Foquet, M.; Craighead, H. G.; Webb, W. W. Zero-mode waveguides for single-molecule analysis at high concentrations. Science 2003, 299, 682-686.

(26) Wenger, J.; Rigneault, H.; Dintinger, J.; Marguet, D.; Lenne, P.F. Single-fluorophore diffusion in a lipid membrane over a subwavelength aperture. J. Biol. Phys. 2006, 32, SN1-SN4.

(27) Samiee, K. T.; Moran-Mirabal, J. M.; Cheung, Y. K.; Craighead, H. G. Zero mode waveguides for single-molecule spectroscopy on lipid membranes. Biophys. J. 2006, 90, 3288-3299.

(28) Miyake, T.; Tanii, T.; Sonobe, H.; Akahori, R.; Shimamoto, N.; Ueno, T.; Funatsu, T.; Ohdomari, I. Real-time Imaging of SingleMolecule Fluorescence with a Zero-Mode Waveguide for the Analysis of Protein-Protein Interaction. Anal. Chem. 2008, 80, 6018-6022.

(29) Liao, D.; Galajda, P.; Riehn, R.; Ilic, R.; Puchalla, J. L.; Yu, H. G.; Craighead, H. G.; Austin, R. H. Single Molecule Correlation Spectroscopy in Continuous Flow Mixers with Zero-Mode Waveguides. Opt. Express 2008, 16, 10077-10090.

(30) Aouani, H.; Mahboub, O.; Devaux, E.; Rigneault, H.; Ebbesen, T. W.; Wenger, J. Plasmonic Antennas for Directional Sorting of Fluorescence Emission. Nano Lett. 2011, 11, 2400-2406.

(31) Larkin, J.; Foquet, M.; Turner, S. W.; Korlach, J.; Wanunu, M. Reversible positioning of single molecules inside zero-mode waveguides. Nano Lett. 2014, 14, 6023-6029.

(32) Goldschen-Ohm, M. P.; Klenchin, V.; Goldsmith, R.; Chanda, B. Single Molecule Ligand Binding FRET at HCN2 Channel Domains in Zero-Mode Waveguides. Biophys. J. 2016, 110, 637a.

(33) Holzmeister, P.; Pibiri, E.; Schmied, J. J.; Sen, T.; Acuna, G. P.; Tinnefeld, P. Quantum yield and excitation rate of single molecules close to metallic nanostructures. Nat. Commun. 2014, 5, 5356.

(34) Pibiri, E.; Holzmeister, P.; Lalkens, B.; Acuna, G. P.; Tinnefeld, P. Single-Molecule Positioning in Zeromode Waveguides by DNA Origami Nanoadapters. Nano Lett. 2014, 14, 3499-3503.

(35) Martin, W. E.; Srijanto, B. R.; Collier, C. P.; Vosch, T.; Richards, C. I. A Comparison of Single-Molecule Emission in Aluminum and Gold Zero-Mode Waveguides. J. Phys. Chem. A 2016, 120, 6719-6727.

(36) Heucke, S. F.; Baumann, F.; Acuna, G. P.; Severin, P. M. D.; Stahl, S. W.; Strackharn, M.; Stein, I. H.; Altpeter, P.; Tinnefeld, P.; Gaub, H. E. Placing Individual Molecules in the Center of Nanoapertures. Nano Lett. 2014, 14, 391-395.

(37) Wenger, J.; Gerard, D.; Dintinger, J.; Mahboub, O.; Bonod, N.; Popov, E.; Ebbesen, T. W.; Rigneault, H. Emission and Excitation Contributions to Enhanced Single Molecule Fluorescence by Gold Nanometric Apertures. Opt. Express 2008, 16, 3008-3020.

(38) Aouani, H.; Mahboub, O.; Bonod, N.; Devaux, E.; Popov, E.; Rigneault, H.; Ebbesen, T. W.; Wenger, J. Bright Unidirectional Fluorescence Emission of Molecules in a Nanoaperture with Plasmonic Corrugations. Nano Lett. 2011, 11, 637-644. 
(39) de Torres, J.; Ghenuche, P.; Moparthi, S. B.; Grigoriev, V.; Wenger, J. FRET enhancement in aluminum zero-mode waveguides. ChemPhysChem 2015, 16, 782-788.

(40) Escobedo, C.; Brolo, A. G.; Gordon, R.; Sinton, D. Optofluidic concentration: plasmonic nanostructure as concentrator and sensor. Nano Lett. 2012, 12, 1592-1596.

(41) Kumar, S.; Wolken, G. G.; Wittenberg, N. J.; Arriaga, E. A.; Oh, S.-H. Nanohole Array-Directed Trapping of Mammalian Mitochondria Enabling Single Organelle Analysis. Anal. Chem. 2015, 87, 1197311977.

(42) Shi, X.; Gao, R.; Ying, Y.-L.; Si, W.; Chen, Y.-F.; Long, Y.-T. A Scattering Nanopore for Single Nanoentity Sensing. ACS Sens. 2016, 1, $1086-1090$.

(43) Kumar, S.; Cherukulappurath, S.; Johnson, T. W.; Oh, S.-H. Millimeter-Sized Suspended Plasmonic Nanohole Arrays for SurfaceTension-Driven Flow-Through SERS. Chem. Mater. 2014, 26, 65236530.

(44) Kumar, S.; Wittenberg, N. J.; Oh, S.-H. Nanopore-induced spontaneous concentration for optofluidic sensing and particle assembly. Anal. Chem. 2013, 85, 971-977.

(45) Martin, W. E.; Srijanto, B. R.; Collier, C. P.; Vosch, T.; Richards, C. I. A Comparison of Single-Molecule Emission in Aluminum and Gold Zero-Mode Waveguides. J. Phys. Chem. A 2016, 120, 6719-6727.

(46) Bharill, S.; Fu, Z.; Palty, R.; Isacoff, E. Y. Stoichiometry and specific assembly of Best ion channels. Proc. Natl. Acad. Sci. U.S.A. 2014, 111, 6491-6496.

(47) Aouani, H.; Mahboub, O.; Devaux, E.; Rigneault, H.; Ebbesen, T. W.; Wenger, J. Large molecular fluorescence enhancement by a nanoaperture with plasmonic corrugations. Opt. Express 2011, 19, 13056-13062.

(48) Wenger, J.; Gérard, D.; Dintinger, J.; Mahboub, O. N.; Bonod, N.; Popov, E.; Ebbesen, T. W.; Rigneault, H. Emission and Excitation Contributions to Enhanced Single Molecule Fluorescence by Gold Nanometric Apertures. Opt. Express 2008, 16, 3008-3020.

(49) Shi, X.; Lim, J.; Ha, T. Acidification of the oxygen scavenging system in single-molecule fluorescence studies: in situ sensing with a ratiometric dual-emission probe. Anal. Chem. 2010, 82, 6132-6138.

(50) Swoboda, M.; Henig, J.; Cheng, H.-M.; Brugger, D.; Haltrich, D.; Plumeré, N.; Schlierf, M. Enzymatic oxygen scavenging for photostability without $\mathrm{pH}$ drop in single-molecule experiments. ACS Nano 2012, 6, 6364-6369.

(51) Henriksen, L.; Grandal, M. V.; Knudsen, S. L. J.; van Deurs, B.; Grøvdal, L. M. Internalization Mechanisms of the Epidermal Growth Factor Receptor after Activation with Different Ligands. PLoS One 2013, 8, No. e58148.

(52) Hellen, E. H.; Axelrod, D. Kinetics of epidermal growth factor/ receptor binding on cells measured by total internal reflection/ fluorescence recovery after photobleaching. J. Fluoresc. 1991, 1, 113128.

(53) Ajo-Franklin, C. M.; Kam, L.; Boxer, S. G. High refractive index substrates for fluorescence microscopy of biological interfaces with high z contrast. Proc. Natl. Acad. Sci. U.S.A. 2001, 98, 13643-13648.

(54) Dixit, P.; Miao, J. Effect of $\mathrm{SF}_{6}$ flow rate on the etched surface profile and bottom grass formation in deep reactive ion etching process. J. Phys.: Conf. Ser. 2006, 34, 577-582. 